\title{
The Wavelet Analysis in the Application of the Traction Substation Feeder Current Analysis
}

\author{
Huang Songwei, Li Huawei, Chang Yujie, Wu Mingli, He Jinghan, Xu Jianjun \\ School of Electrical Engineering, \\ Beijing Jiaotong University \\ Beijing 100044, China \\ e-mail: 12291030@,bjtu.edu.cn
}

\begin{abstract}
This paper is with the purpose of catastrophe point identification and comparison between wavelet analysis and Fourier analysis on their effects of filtering, and apply it into the analysis of traction substation feeder current. First, we assess the differences between wavelet analysis and Fourier analysis by doing these two analyses to the ideal noise signal; and then we study the best conditions of using wavelet analysis and wavelet filtering conditions through the experiment using MATLAB and wavelet toolbox to explore the methods; last, we adopt the method that combining FFT analysis and wavelet analysis to analyze the traction substation feeder current. The simulation shows that wavelet analysis can recognize the breakpoints' location of signal, while Fourier analysis can't. The effects of wavelet filtering are better than Fourier analysis, while FFT can analyze the content of harmonic wave in signal. This paper also provides an example for fusion of science and education.
\end{abstract}

Key words: wavelet analysis; Fourier analysis; filtering; MATLAB wavelet toolbox; feeder current.

\section{INTRODUCTION}

In traction substation, the feeder current has great effect, for example, feeder current can be used to estimate current transformer capacity utilization, calculate the transformer capacity and catenary wire fever, used for setting of relay protection devices, etc. [1]. Feeder current plays a great role in guaranteeing trouble-free equipment continuing to run normally, and to maximize continuous power supply to protect the safety of trains, etc. Thus the eigenvalues of the feeder current, such as noise, harmonic component and fundamental wave amplitude and so on has become the focus of the research. At present, many researchers use the Fourier analysis to analyze the feeder current harmonic signals and filter wavelet according to Fourier analysis of the results. The advantage is Fourier can indeed acquire good analysis of the different harmonic component [2], but cannot have very good effect on those of high frequency, localization of the noise signal. To solve this problem, this paper combined with Fourier and wavelet analysis to provide a better way for the feeder current analysis.

This paper first compared the Fourier transform and wavelet transform. Then we studied the application of wavelet analysis under de-noising conditions. Finally we used the methods of Fourier analysis and wavelet analysis to analyze and filter feeder current signal and compared the Fourier filtering with wavelet filtering from the results.

\section{COMPARISON OF WAVELET ANALYSIS AND FOURIER ANALYSIS}

Fourier analysis is a method which decomposes the signal into different frequency components, and then analyzes these components. But the wavelet analyzes by scale transformation. On the analysis of low frequency signal, it uses stretching wavelet and long-time window, and the analysis of the high frequency signal, it uses compressed wavelet and short-time window, ultimately achieve high frequency time segment, the low frequency in the frequency segment, it can automatically adapt to the requirements of time-frequency signal analysis [3].

The following will be based on Fourier transform and wavelet transform formula to contrast to the discussion of the difference between the two.

$$
F(\omega)=\int_{-\infty}^{\infty} f(x) e^{j \omega x} d x
$$

$W T_{f}(a, b)=|a|^{-1 / 2} \int_{-\infty}^{\infty} f(t) \overline{\left(\frac{t-b}{a}\right)} d t, \quad a \neq 0$

Among them $\psi_{a, b}(t)$ is little wave function, $\psi_{a, b}(t)=|a|^{-1 / 2} \psi\left(\frac{t-b}{a}\right)$.

Equation (1) is Fourier transform formula, (2) is Wavelet transform formula [4]. There are four differences between the two transform formulas.

1) Fourier transform convert signal from time domain $t$ to frequency domain, which is one-dimensional transformation [5], while wavelet transform convert time domain into a formula affected by both two factors that are time domain $\mathrm{u}$ and scale $\mathrm{s}$, which is two-dimensional transformation [6]. What's more, two factors can express much more information than that by one. Wavelet decomposes on different dimensions, which equals of converting under different $\mathrm{u}$, in which every scale has its own detail information to bring more amounts of information to signal analysis [7].

2) The blocks of Fourier transform is sine wave or cosine wave, which means Fourier transform decompose signals into overlays of sine wave and cosine wave in 
different frequency and sine wave is periodic and infinitely long, which has good pertinence to approximately periodic signals. However signals are not continuous and steady on most occasions, signals always have some tiny mutation components which is hard to be expressed in sine wave or cosine wave. While the block of wave transform is wave whose features are having none zero value only in a finite region and it has orthogonality and greater selectivity, and in base of different wave, we can choose different waves to make the superposed wave more similar with original signal by superimposition.
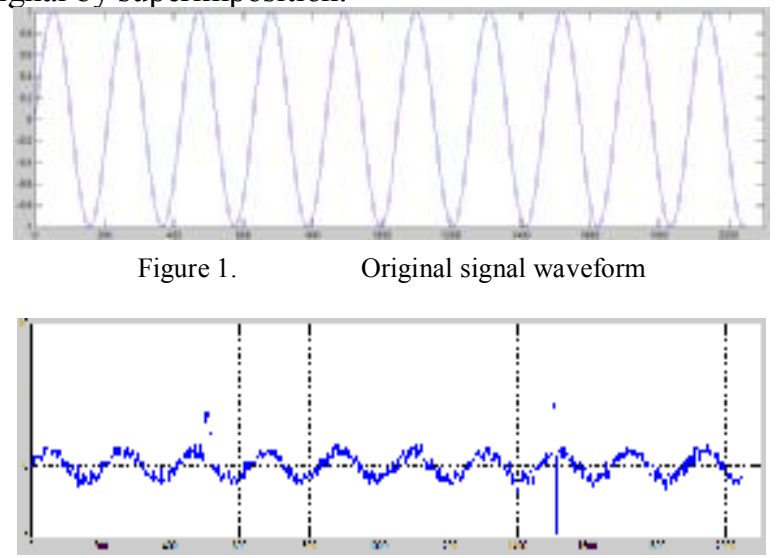

Figure 2.

Signal contains noise

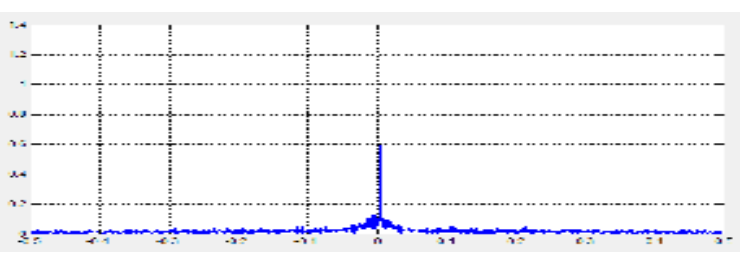

Figure 3.

Spectrum diagram

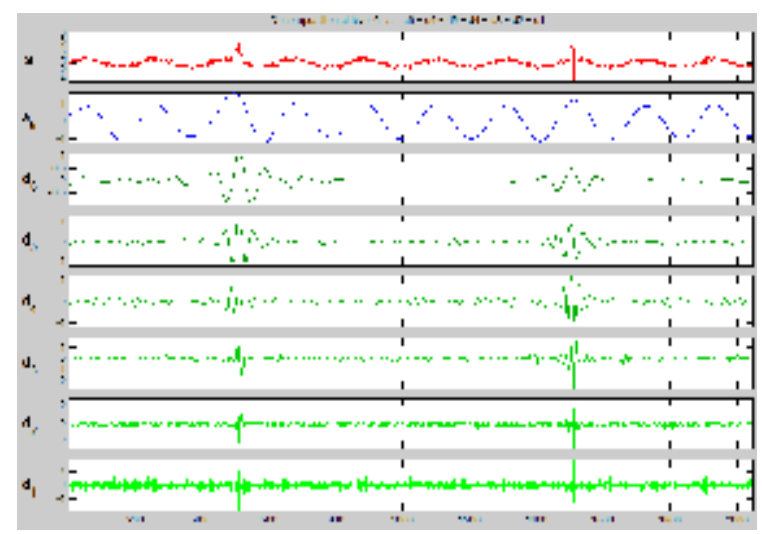

Figure 4.

Waveforms of six layers after wavelet transform

Fig. 1 is an original signal, and it will get a noisy signal as shown in Fig. 2 when overlap ordinary noisy signal and two localized noisy signals on it. Analysis this signal with FFT to obtain its spectrogram, which is shown as Fig. 3, from which we can know that is a sine signal with 0.0048 $\mathrm{Hz}$ in frequency and 1 in amplitude, but could not recognize whether it has localized noisy signal. However, when we use wavelet to analysis, when we choose wavelet Db34 to decompose this signal into 6 layers in this example, we can obtain a set of waveform diagram, as shown in Fig. 4. In Fig. 4, a6 is decomposed similar signal, and $\mathrm{d} 1$ to $\mathrm{d} 4$ are detail signals, and similar signal is sine signal with 1 in amplitude. From its high frequency sector, we can recognize that it has greater unusual change around 500 and 1500. So wavelet analysis can express local change, owing to changeability of wavelet basis function block.

3) Though Fourier transform which decomposes signal on frequency domain can show the signal contained different frequency clearly, but it can't locate the noisy signal, while wavelet analysis can locate the noisy signal from those time domain diagram when wavelet analysis is decomposing signal under different scale s to make similar signal and detail signal, which is equivalent to decompose under different frequency [8]. As shown in Fig. 4, it can indicate that the local signal and abrupt noise are at 500 and 1500 on $\mathrm{x}$-coordinate.

4) Using wavelet analysis can distinguish the noise information from signal more correctly, as shown in above case, it can only be known the range of signal frequency when using Fourier analysis and then construct filter to filtrate it. While when noise is local signal and part of frequency is during the frequency range of the signal, the noise can't be filtered out, but after wavelet analysis, in every layer of decomposition, noise signal and scale of local signal can be localized. Then choose a threshold for every layer, and construct filter to filtrate it, in order to wipe out noise signal and localized signal, so that it can filter the signal more correctly. Fig. 5 shows the waveform obtained by filtering using low-pass filter after Fourier transform directly, and Fig. 6 shows the waveform obtained by setting a threshold for every decomposed layer to filtrate out noise signal after using wavelet analysis. It is obvious that filtering after wavelet analysis can wipe out the noise signal better when comparing the two figures.

To sum up, using wavelet analysis to analyze signal has better effect.

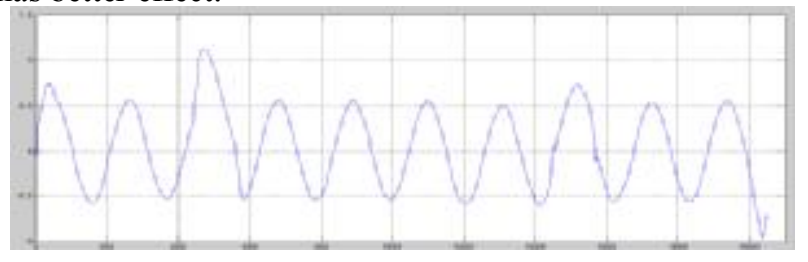

Figure 5. Filter directly after Fourier analysis

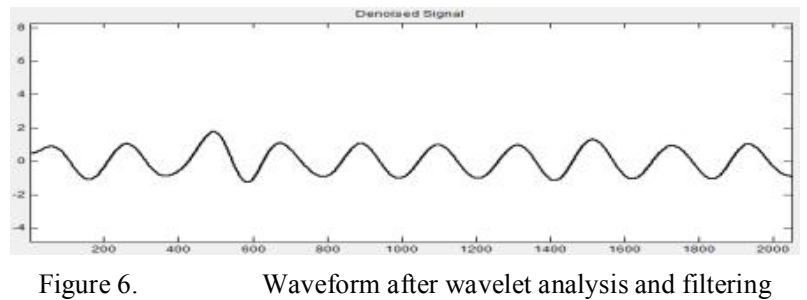

\section{THE METHOD OF WAVELET DENOISING CONDITION SELECTION}

\section{A. Wavelet Basis Function Selection}

Choosing a suitable wavelet basis function will have a great impact on the precision filter; we use the error between the results of wavelet analysis and the theoretical value to select wavelet basis.

Generally, there are two methods to choose wavelet basis function. The first kind uses wavelet toolbox to 
choose. For a noisy signal, we can filtering it directly by toolbox to determine preliminarily effect of which wavelet filtering is better, which method that is rough.

The second method is comparing several indexes to choose suitable wavelet basis function, which indicates that the greater the signal to noise ratio and the smaller the root mean square error are, then more suitable the wavelet basis function is.

$$
\text { RMSE }=\sqrt{\frac{2}{n} \sum_{n}[f(n)-f(n)]^{2}}
$$

$$
\mathrm{SNR}=10 \times \log _{10}\left(\text { Power }_{\text {gignal }} / \text { Power }_{\text {noise }}\right)
$$

$$
\text { Power }_{\text {signal }}=\frac{1}{n} \Sigma_{n} f^{2}(n)
$$

$$
\text { Power neise }=\frac{1}{n} \Sigma_{n}[f(n)-f(n)]^{2}
$$

Equation (3) is the RMS error formula, $f(n)$ is the original signal; $f(n)$ is a reconstruction signal which is in a certain scale; $\mathrm{n}$ is the length of the signal. Power sig nal is the original signal power. Power notse is the noise power [9].

TABLE I. RMS ERROR AND THE SIGNAL-TO-NOISE RATIO OF SIGNAL WHICH USES DB WAVELET FILTERING

\begin{tabular}{|c|c|c|}
\hline $\begin{array}{c}\text { Wavelet basis } \\
\text { function }\end{array}$ & RMS error & SNR \\
\hline $\mathrm{db} 3$ & 0.2533 & 10.3775 \\
\hline $\mathrm{db} 4$ & 0.2577 & 10.2273 \\
\hline $\mathrm{db} 5$ & 0.2632 & 10.0417 \\
\hline $\mathrm{db} 6$ & 0.2261 & 11.3613 \\
\hline $\mathrm{db} 7$ & 0.2307 & 11.1874 \\
\hline $\mathrm{db} 8$ & 0.2402 & 0.8361 \\
\hline $\mathrm{db} 9$ & 0.2249 & 11.4098 \\
\hline $\mathrm{db} 32$ & 0.1586 & 14.4410 \\
\hline
\end{tabular}

We use $\mathrm{db}$ wavelet basis function wavelet filtering, the signals are analyzed to calculate the RMS error and the signal-to-noise ratio of data as shown in Table 1. Through the comparison, we can find that after db32 wavelet filtering, SNR is maximum, and root mean square error is minimum. Thus, for the noise signal, db32 filtering effect is the best.

Above all, we can firstly use wavelet toolbox to select suitable wavelet roughly, and then compare the signal-tonoise ratio and root mean square by programming to select the most suitable wavelet.

\section{B. the Number of Decomposition Layers}

Different number of decomposition layers will affect different filtering effect. We can use wavelet toolbox to do preliminary experiment to choose the number of decomposition layers. We decompose the signal from 1 to 10 layers, and then reconstruct the signal, see the effect of different decomposition layer filter, to choose appropriate decomposition layers. Through the experiment, we get the following conclusion: when the decomposition layers for five or six layers, it has the best de-noising effect. When decomposition layers are too much, it will lead to the filter graph distortion.

\section{C. the Choice of Threshold}

In wavelet analysis, the choice of the threshold is very important; the threshold directly determines the quality of the signal de-noising. In the signal filtering, we can use programming for filter, and we can also use wavelet toolbox to filter.

In MATLAB programming, it has the following four threshold selection: (1) "rigrsure", (2) "heursure", (3) "sqtwolog", and (4)"minimaxi" [10]. And threshold reset generally has three conditions: (1) no need to be adjusted (recorded as "one"); (2) only being adjusted when the wavelet decomposition of the first layer of the threshold (remember "SLN"); (3) the threshold to be adjusted in each layer (remember "MLN").

In the project, the choice of the threshold need to adjusting according to the actual situation, when the signal is contained in the local mutation signal, choose the threshold adjustment coefficient of each layer, it has better de-noising effect.

When using wavelet toolbox for filtering and analysis, by using wavelet toolbox to decompose the signal into different layers. First, analyze the signal of each layer, if there has abnormal mutated in a layer of detail component part, set the threshold value to remove the mutation.

\section{THE ANALYSIS OF THE FEEDER CURRENT}

Harmonic produced by electric locomotive load will not only cause the decline of power quality, but also make electrical equipment for heating. So it is very important to detect circuit of harmonic signal; in circuit failure, meanwhile, if you want to detect circuit fault transient components, you have to pinpoint the weak fault signal, it has to give a method to accurately analyze the signal. To meet the requirements of the above analysis, this paper combines the Fourier and wavelet analysis to form a filtering method with high accuracy which not only can locate fault point, but also can analyze harmonic.

\section{A. Analysis of Harmonic Wave of Feeder Current and Detail Signal}

As shown in Fig. 7 is a feeder current signal of traction substation which is measured in some conditions. To know that what frequency harmonic component is contained in the feeder current, we use FFT to analyze it, and we can get the spectrogram as shown in Fig. 8. According to the spectrogram we can see the signal contains $50 \mathrm{~Hz}$ fundamental wave and $150 \mathrm{~Hz}$ and $150 \mathrm{~Hz}$ and $150 \mathrm{~Hz}$, $450 \mathrm{~Hz}$ and $550 \mathrm{~Hz}$ and $650 \mathrm{~Hz}$ and $750 \mathrm{~Hz}$ harmonic. Measuring the harmonic signal can provide conditions for 
hardware filtering and can also provide hardware setting up different filter to eliminate harmonic.

For the analysis of feeder current signals in detail, we can use wavelet analysis to implement. We can choose suitable wavelet by wavelet toolbox, through experiments, we can know that choose $\mathrm{db} 34$ wavelet is more appropriate; Using wavelet toolbox to decompose the signal into 6 layers, and then we can get the signal that can be obtained to estimate the signal and detail signal as shown in Fig. 9.

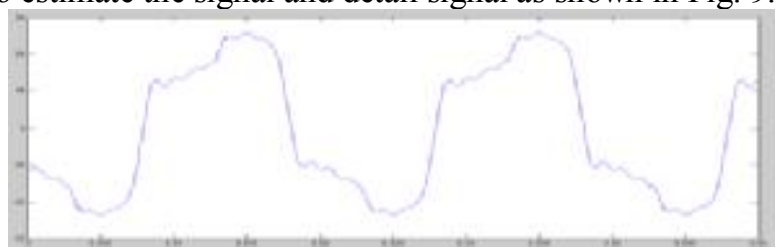

Figure 7.

Waveform of feeder current signal

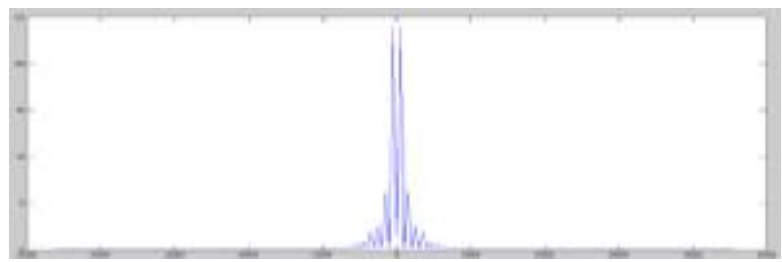

Figure 8.

Spectrum diagram of feeder current

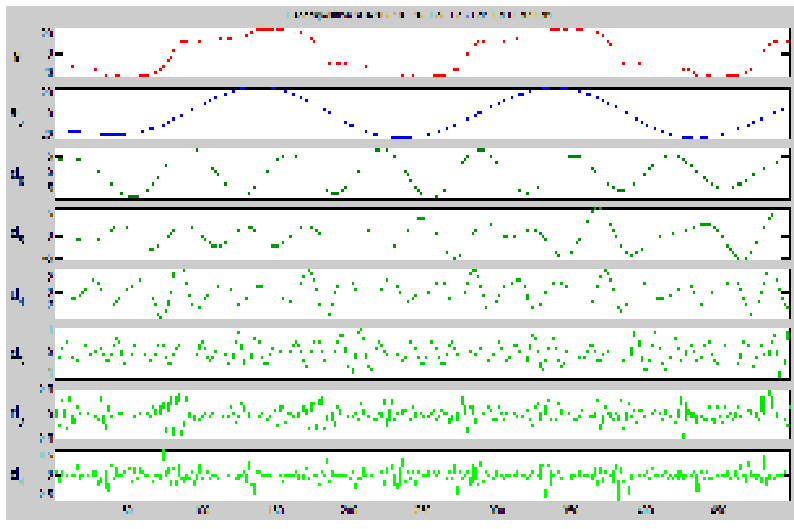

Figure 9.

Waveform of six layers after doing wavelet analysis to feeder current

As we can see from Fig. 9, the approximation of the decomposed signal is close to the fundamental wave current signal, and detail signal containing high frequency signal and harmonic signals. And through the picture, we can see detail signal information in each layer, providing clearer threshold selection for filtering.

\section{B. Contrast of FFT and Wavelet the Feeder Current Filter}

If we only put the high frequency signal filtering from the feeder current signal, we can get the waveform like Fig. 10. If you want to get rid of all of the harmonic signals, you can do that by setting the threshold value of each layer, filtering out the approximate signals outside of all the details, and then you can get base wave signal waveform, as shown in Fig. 11. Using FFT to analyze the signal and then will be filtering by calling function, we can get the waveform which is de-noised as shown in Fig. 12.

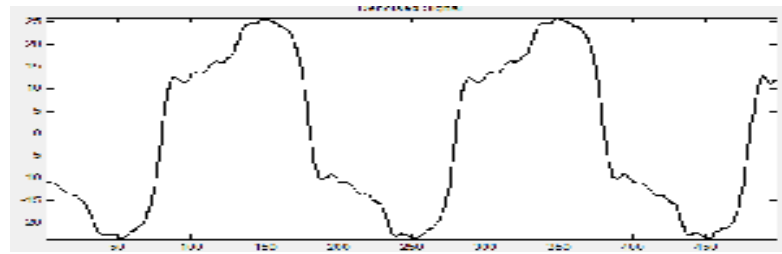

Figure 10. Signal waveform after filtering high-frequency noise

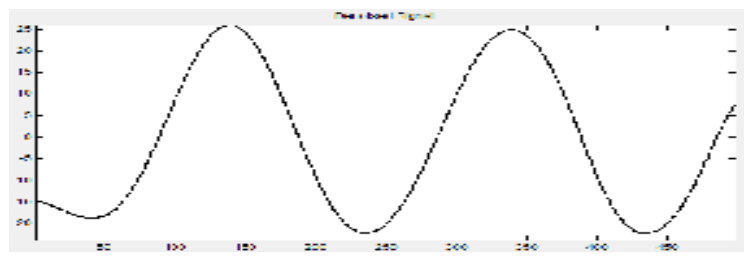

Figure 11 .

signal waveform after filtering harmonic wave signal out

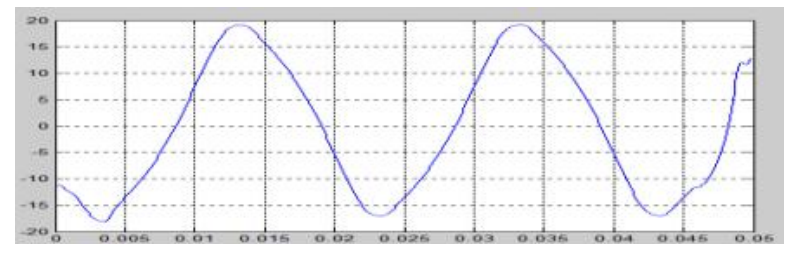

Figure 12 .

FFT filtering

Comparing Fig. 11 with Fig. 12, we can know that the waveform after the feeder current signal wavelet filtering is smooth, and is similar with the fundamental wave signal. Though FFT filtered harmonic wave out as well, the waveform is a bit out of shape.

So we can make analysis of the similarities and differences between filtering by FFT and wavelet, as shown in the following table.

TABLE II. THE SIMILARITIES AND DIFFERENCES BETWEEN THE WAVELET FIL TERING AND FFT FILTERING

\begin{tabular}{|l|l|l|}
\hline & The wavelet filtering & FFT filtering \\
\hline $\begin{array}{l}\text { Analysis } \\
\text { before } \\
\text { filtering }\end{array}$ & $\begin{array}{l}\text { Layer the signal into } \\
\text { superposition of } \\
\text { approximate signals and } \\
\text { detail signals }\end{array}$ & $\begin{array}{l}\text { Analyze frequency } \\
\text { components of signal } \\
\text { by spectrogram of signal. }\end{array}$ \\
\hline $\begin{array}{l}\text { Threshold } \\
\text { selection }\end{array}$ & $\begin{array}{l}\text { Analyze signal amplitude } \\
\text { of each layer and then set a } \\
\text { threshold value separately } \\
\text { for each layer }\end{array}$ & $\begin{array}{l}\text { analyze the spectrum } \\
\text { diagram to obtain the } \\
\text { major frequency and select } \\
\text { the appropriate threshold }\end{array}$ \\
\hline Filtering & $\begin{array}{l}\text { Filter signal of different } \\
\text { frequency band to choose } \\
\text { the required parts }\end{array}$ & $\begin{array}{l}\text { Poor selectivity, it } \\
\text { may filter out effective } \\
\text { signal part some can't } \\
\text { filter out noise signal }\end{array}$ \\
\hline
\end{tabular}

In conclusion, the wavelet filter has better effect on signal de-noising effect.

\section{CONCLUSIONS}

This paper introduced method of analysis for feeder current which combines Fourier analysis and wavelet analysis, on the basis of filtering of substation's feeder current and necessity of analysis. This paper also shows that FFT can be used to analyze for information of feeder current's harmonic wave, and wavelet analysis is used to obtain detail information of feeder current and localized 
fail signal in this paper, and the effect of wavelet analysis is better than that of Fourier analysis. This paper also analyses their own advantages of wavelet analysis and Fourier analysis by the comparison of Fourier analysis and wavelet analysis theoretically. What's more, this paper provides the method to choose filtering condition more correctly by using wavelet-box of MATLAB and MATLAB programming.

\section{REFERENCES}

[1] Q. Z. Wan, M. L. Wu, J. Y. Chen, Z. J. Wang, and X. Y. Liu, "Probability Distribution of Feeder Current of Traction Substation," Journal of Southwest Jiaotong University.

[2] P. Sun, "Power Quality Measurement Method Based on The combination of The Wavelet and Fourier transform," Programmable Controller \& Factory Automation, vol. (5), 2015, pp. 78-82.

[3] A. G. Song, W. B. Liu, and A. M. Wang, Test Signal Processing And Analysis. Beijing: China Machine Press,2007
[4] J. T. Chui, An Introduction to Wavelets. Pennsylvania: Academic Press, 1992

[5] Y. M. Zhong, S. R. Qin, and B. P. Tang, "Uniform Mathematics Model of The Fourier Transform And The Wavelet Transform," Chinese Journal of Mechanical Engineering, vol. 38(11), 2002 pp.36-41.

[6] M. Yang, Z. W. Zhang, H. Q. Sun, and Y. D. Zhang, "The Comparison of Wavelet and Fourier Analysis and Their Application to Fault Diagnosis." China Measurement Technology, vol. 31(2), 2005, pp.58-61.

[7] J. B. Jiang, X. X. Liu, and D. M. Wang, "Application of FFT and Wavelet Transform to Measurement of Harmonics in Power System," High Voltage Engineering, vol. 31(11), 2005, pp.85-87.

[8] W. G. Hao, C. F. Ding, and N. Liang, "Comparison of Wavelet Denoise and FFT Denoise," Electric Power Science And Engineering, vol. 27(3), 2011,pp.59-61.

[9] K. J. Tao, and J. J. Zhu, "A Comparative Study on Validity Assessment of Wavelet De-noising," Journal of Geodesy and Geodynamics, vol. 32(2), 2012, pp.128-133.

[10] H. J. Yu, "Selection of A Wavelet Noise-reduction Threshold," Journal of Shaoxing Univerity, vol. 24(9) ,2004, pp.34-38. 